Artículos / Articles 


\title{
Realidad y ciencia en el realismo analítico de Talcott Parsons y el constructivismo operativo de Niklas Luhmann/
}

\author{
Reality and Science in Talcott Parsons's Analytical Realism and Niklas
}

Luhmann's Operative Constructivism

\author{
*Pedro Giordano \\ Instituto de Investigaciones Gino Germani. Facultad de Ciencias Sociales. Universidad de Buenos Aires. \\ República Argentina/Argentine Republic \\ pedrogiordan083@yahoo.com.ar \\ Recibido / Received: 19/09/2016 \\ Aceptado / Accepted: 08/06/2017
}

RESUMEN

El presente ensayo compara las posturas epistemológicas con las que Talcott Parsons y Niklas Luhmann encaran el problema de la adecuación entre conocimiento y realidad, para luego indagar la concepción de ciencia que se desprende de sus respectivas decisiones. A su vez, el modo en que cada uno emplea la noción de sistema constituye un factor de análisis clave, ya que permite entrelazar ambas problemáticas. Para realizar la tarea, primero se examina la epistemología realista analítica de Parsons con foco en la relación entre realidad y ciencia; luego, se realiza el mismo ejercicio con la epistemología constructivista operativa de Luhmann; en tercer lugar, se comparan ambas posturas en busca de similitudes y diferencias que permitan iluminar la naturaleza del vínculo teórico entre ambos autores; finalmente, se concluye que el constructivismo operativo puede leerse en clave de una propuesta que pretende superar las aporías intrínsecas al realismo analítico.

Palabras clave: Realidad, ciencia, sistema, realismo analítico, constructivismo operativo.

\section{ABSTRACT}

This paper compares Talcott Parsons' and Niklas Luhmann's epistemological positions and their understanding of science. For our analysis, their conceptualization about "systems" appears as a key factor since it intertwines both issues. In order to do so, first, we explore Parsons' "analytical realism" focusing on the relationship between reality and theory; second, we explore Luhmann's "operative constructivism"; third, both positions are compared in order to highlight which similarities and differences could shed light on the relations between the two authors; last, we argument towards an interpretation of Luhmann's "operative constructivism" as an improvement of Parson's "analytical realism" limitations.

Keywords: Reality, science, system, analytical realism, operative constructivism.

\footnotetext{
*Autor para correspondencia / Corresponding author: Pedro Martín Giordano. Instituto de Investigaciones Gino Germani. Pte. J. Uriburu 950, 6. ${ }^{\circ}$ piso, of. 1. Buenos Aires, Argentina.

Sugerencia de cita / Suggested citation: Giordano, P. (2018). Realidad y ciencia en el realismo analítico de Talcott Parsons y el constructivismo operativo de Niklas Luhmann. Revista Española de Sociología, 27 (1), 9-24.

(Doi: http://dx.doi.org/10.22325/fes/res.2018.1)
} 


\section{INTRODUCCIÓN}

La pretensión de alejarse del pensamiento social europeo tradicional constituye uno de los pilares de la obra de Niklas Luhmann. Sin embargo, y pese a esa voluntad manifiesta, hay quienes encuentran elementos suficientes para ligar su producción con la tradición clásica. Sobre la base de que el propio Luhmann entiende que lo característico de una teoría clásica consiste en amalgamar un conjunto de afirmaciones, obsoletas en cuanto a sus respuestas pero actuales en lo relativo a las preguntas que plantean, se sostiene que en el intento de explicar la sociedad en carácter de unidad histórica, de describir procesos de estructuración y cambio social, de deslindar la dimensión subjetiva de los fenómenos normativos que la trascienden y se le imponen, pueden observarse ciertas continuidades con la teoría sociológica (Castro Muzupappa, 1997). Otros conciben esta relación en términos paradójicos: por un lado, el diagnóstico de crisis teórica en el que se encuentra la sociología conduce a Luhmann a desechar su marco conceptual y optar por la obtención de insumos procedentes de disciplinas foráneas; por el otro, su permanente adscripción a la sociología constituye un dato relevante al momento de alinear su obra en las filas de la discusión clásica (Vanderstraeten, 2002).

En ambos casos, se afirma que Luhmann guía sus investigaciones tomando las preguntas de la sociología, pero cuando elabora sus respuestas, desplaza la mira hacia otras áreas de investigación. En sus propias palabras, el abandono del esquema clásico se debe a su incapacidad de avanzar hacia la elaboración de un marco de referencia abstracto, aplicable a la observación de la sociedad y sus sistemas constituyentes. Aduce que la proliferación de investigaciones empíricas (con éxito demostrable), acrecienta el conocimiento aunque no aporta a la configuración de una teoría capaz de fundamentar el campo específico de su objeto y lograr la unidad de su disciplina: "la resignación ha ido tan lejos que ya ni siquiera se hace el intento" (Luhmann, 1998b: 7). En este marco, y para superar el estado crítico, propone el desarrollo de una teoría autorreferencial con pretensiones universalistas fundada en los lineamientos de la Teoría de Sistemas, pues- to que la introducción de sus categorías contribuiría a promover cambios profundos y revolucionarios (en términos kuhnianos) a lo largo y a lo ancho del campo científico (Luhmann, 1998b).

En el abierto enfrentamiento con la tradición sociológica, no todos sus referentes corren la misma suerte: entre 1960 y 1961, Luhmann cursa una serie de seminarios sobre sociología dictados por Talcott Parsons. Además de mantener una comunicación intelectual directa con el maestro y de verse seducido por su ambición teórica, la estancia en Harvard le brinda la posibilidad de apreciar cómo se retoma críticamente la tradición europea en este centro creador de teoría sociológica (Izuzquiza, 2008). Al referirse a los textos de Parsons, afirma que la amplitud de un proyecto caracterizado por la codificación del saber de los clásicos y la elaboración del entendimiento conceptual de la acción, hacen de su propuesta la única teoría sociológica sistemática existente hasta el momento (Luhmann, 1990a). Sin ser ajeno al alud crítico que desde fines de los años cincuenta sacude todos los principios de la Teoría General de la Acción de Parsons, entiende que ninguna de esas voces logra acercarse a su nivel de abstracción. Para no cometer el mismo error, retoma explícitamente el objetivo parsoniano de formular una teoría universal de la sociología (Luhmann, 1998b). Hoy en día, hay acuerdo en que toda su obra se halla traspasada por la referencia obligatoria a Parsons (Nafarrate, 2007); y además, que debido a su ambición de construir una teoría general de los sistemas sociales — principalmente de la sociedad moderna-, con profundidad y consistencia demostradas, la teoría de la sociedad de Luhmann es la única producción en situación de reclamarse heredera del pensamiento parsoniano (Fuchs, 2001).

Por estas razones, el vínculo entre ambos autores constituye un tema de investigación frecuentado por la sociología contemporánea. Una vez descartadas las primeras interpretaciones que sólo vieron en Luhmann el intento de actualizar el esquema de Parsons según las innovaciones más relevantes de la época, creció la preocupación por desentrañar los núcleos temáticos que los entrelazan, tarea para la cual se formularon diversos ejes de comparación. Solo para citar algunos, hay quienes ponen el foco 
de atención en la manera en que cada uno aborda el problema de la doble contingencia (Vanderstraeten, 2002); en las diferencias de la noción de sistema que elaboran (Camou, 1997); en la consideración de los medios de intercambio como el origen de los medios de comunicación simbólicamente generalizados (Chernilo, 1999; García Ruiz, 1997; Mascareño, 2009); 0 en el esclarecimiento del pasaje desde una metodología estructural-funcionalista a una funcionalista-estructural ${ }^{1}$ (Izuzquiza, 2008).

Con el objetivo de contribuir a la comprensión del vínculo entre ambos autores, este ensayo propone abordar un área escasamente revisada por la bibliografía especializada: se trata de analizar, en clave comparativa, las posturas epistemológicas con las que cada uno encara el problema de la adecuación entre conocimiento y realidad para, en seguida indagar la concepción de ciencia que se desprende de sus respectivas decisiones. A su vez, la forma en que cada autor utiliza la noción de sistema y los diversos modos en los que la emplean, constituye un factor clave del análisis, pues permite enlazar ambas problemáticas. Para ello, en el primer apartado se examina la epistemología realista analítica de Parsons con énfasis en la relación entre realidad y ciencia; en el segundo, se realiza el mismo ejercicio con la epistemología constructivista operativa de Luhmann; en el tercero, se comparan ambas posiciones; finalmente, en las conclusiones se presentan los resultados obtenidos.

Antes de comenzar, cabe aclarar que el presente trabajo es de carácter teórico y se acopla a la persistente discusión acerca de la especificidad de la sociología, su objeto de estudio y sus presupuestos epistemológicos. Se trata de entender cómo interpretan dichas temáticas los autores seleccionados, sobre todo porque Parsons postula la inmadurez de la disciplina y elabora una teoría que pretende su-

1 En cuanto a los avatares del vínculo de Parsons con el estructural-funcionalismo resalta que, si bien ayudó a expandir la corriente en el campo de la sociología, su postura presenta una serie de divergencias con respecto a la de sus padres fundadores. Para un tratamiento sobre el tema se recomienda ver (Lagunas, 2016). Por su parte, la crítica de Luhmann a esta tradición y su propuesta de transformarla en un funcionalismo-estructural se encuentra en (Niklas Luhmann, 1973a, 1973b). perarla, mientras Luhmann — varias décadas después - lleva a cabo una evaluación crítica acerca del estado de la sociología y la sitúa a nivel teórico. Ambos consideran que el modo de fortalecerla se liga a la construcción teórica, empresa que llevan adelante abrevando en áreas aledañas.

En esa dirección, resulta pertinente profundizar en sus concepciones de realidad y ciencia, un ejercicio que implica la revisión de sus premisas epistemológicas con el propósito de aportar a la clásica disputa acerca de los límites de la sociología con respecto a otros campos del saber.

\section{EL REALISMO ANALÍTICO DE TALCOTT PARSONS}

Dada su pretensión de construir una Teoría General de la Acción (en adelante TGA) de carácter válido dentro del campo científico, uno de los principales problemas al que se enfrenta Parsons es al de la referencia empírica de su propia teoría: debe precisar un enfoque epistemológico que defina el estatus de los conceptos respecto de la realidad. Será el realismo analítico, la postura epistemológica que lo acompañará, con escasas modificaciones, a lo largo de su carrera intelectual. En su primer gran obra, La Estructura de la Acción Social (Parsons, 1971a, 1971b) presenta los principales lineamientos de una postura que consta de dos partes: es realista porque considera la existencia de un mundo externo de realidad (un orden fáctico), que no es creación humana, ni puede ser reducida a términos de un orden ideal, por ejemplo, el de un sistema filosófico. En segundo lugar, es analítico, porque los elementos de los que se vale no son la realidad directa, sino una representación ideal de ella. En el marco de esa orientación, Parsons alega que un sistema de teoría científica es una representación adecuada, no literal, de la realidad externa: su orden lógico se corresponde con el orden fáctico (la correcta denominación a este tipo particular de interrelación entre sistema teórico y realidad es la de funcionalidad). Ese punto de partida acarrea importantes consecuencias para el resto de la teoría. En primera instancia, sobresale la distinción entre fenómenos y hechos: mientras los primeros son entidades concretas realmente existentes, los 
segundos son afirmaciones empíricamente verificables acerca de los fenómenos, en términos de un esquema conceptual. La distinción, según la cual se afirma que los hechos no son fenómenos en sí sino enunciados acerca de ellos, conduce la actividad científica a un nuevo plano, el del mundo transfenoménico, o el de la pura abstracción. En ese sentido, un sistema de teoría científica se compone de hechos que no describen completamente los fenómenos, sino sólo su parte relevante según el esquema conceptual. Como el propósito de la ciencia no radica en describir plenamente todos los sucesos concretos, sino sólo aquellos previamente seleccionados mediante el esquema conceptual ${ }^{2}$, el realismo se vuelve analítico justamente porque se sitúa en un nivel intermedio de abstracción, entre la pura empiria y la irrealidad (Parsons, 1971a, 1971b).

A su vez, el realismo analítico ${ }^{3}$, constituye una intersección que pretende evitar los errores ficcionalistas y empiristas ${ }^{4}$ : se aleja de los primeros ${ }^{5}$, pues entiende que algunos de los conceptos generales de la ciencia no son pura ficción construida por el observador, ya que en algún punto logran captar aspectos del mundo externo objetivo; se enfrenta a los segundos cuando indica que dichos conceptos

2 En este punto, se reconoce heredero del pensamiento de su profesor L. J. Henderson, para quien toda observación, desde la más simple (la del sentido común) a la más compleja (la de la ciencia), pasa por el filtro de un esquema conceptual (Parsons, 1971a).

3 Uno de sus principales críticos de habla hispana, José Almaraz (1981), denomina nominalismo moderado a esta postura, ya que, por un lado presenta rasgos nominalistas, porque entiende que es el científico quien reconstruye la realidad, por intermedio de símbolos conceptuales que seleccionan aspectos de ella; pero a su vez, el nominalismo se modera al trabajar con abstracciones analíticas, que nos son consideradas ficciones útiles sino representaciones adecuadas.

4 La crítica a esta corriente se asienta, principalmente en lo que Withehead denomina la falacia de lo concreto mal ubicado, que implica creer que la teoría es un reflejo especular de la realidad, que entre teoría y empiria se verifica una coincidencia punto por punto (Parsons, 1971a; Whitehead, 1985).

5 Principalmente, se refiere a los tipos ideales de Max Weber, los cuales no reflejan la realidad sino que representan útiles ficciones (Parsons, 1971a). no se corresponden de manera directa con los fenómenos concretos, sino solo con elementos de ellos, analíticamente separables de otros elementos (Parsons, 1968). Así, su posición filosófico-ontológica no sólo evalúa la existencia real de los fenómenos; además, se interroga acerca de la propia veracidad de lo real ${ }^{6}$.

Una vez establecida la relación entre ciencia y realidad, Parsons concentra todos sus esfuerzos en el desarrollo científico de la sociología. Su punto de partida informa acerca del estado de inmadurez en el que se encuentra, en comparación con las ciencias naturales ${ }^{7}$; el indicador de esa situación es el inferior nivel de desarrollo de su teoría sistemática, sobre todo, las especies y grados de integración lógica de los diferentes elementos que constituyen su esquema conceptual (Parsons, 1967). Por tanto, su proyecto se orienta a llenar ese vacío, de modo de otorgar a la disciplina rasgos de madurez. En esa dirección, y en primer lugar, precisa conceptualmente los elementos constitutivos de su teoría sistemática, tarea que comienza con la formulación del marco de referencia, encargado de construir el campo más general de categorías en cuyos términos el trabajo empírico científico cobra sentido. Puesto que toda

6 Sobre el vínculo entre la postura epistemológica y la ontológica, Savage (1999) comenta que la tesis principal del realismo analítico en cuanto a la realidad es que el sistema de teoría científica realista y analítico aprehende la realidad; es decir que hay correspondencia entre orden lógico y orden natural del mundo real. Subyace a esta postura una ontología definida: la concepción epistemológica se asienta sobre la base de que la realidad es un sistema regular y ordenado que puede ser representado adecuadamente por la teoría. El problema radica en que "la concepción ontológica no puede justificarse por la epistemología" (Savage, 1999: 73). Si la ontología constituye la condición lógica de la epistemología, no puede ser demostrada por ésta, porque se cae en un argumento circular donde la concepción ontológica se justifica a sí misma. El realismo analítico, entonces, no justifica teóricamente que el mundo es ordenado, condición de posibilidad de su aprehensión; al no hacerlo, sostiene una concepción dogmática, carente de comprobación.

7 En el horizonte destacan la mecánica analítica y la matemática de cálculo diferencial (Parsons, 1967). 
ciencia inicia con un proceso de observación que establece los límites de su futura actividad, el instrumento adecuado para realizar la operación es el marco de referencia, ya que se establece como instancia configuradora del campo específico de la observación del sistema teórico, al tiempo que distingue lo que debe ser explicado de aquello que carece de relevancia teórica: en cuanto elaboración conceptual, cumple la función de describir y constituir el objeto científico; es decir que, guía lógica y metodológicamente los conceptos y proposiciones que forman parte del sistema teórico. Un sistema teórico, en cambio, es un cuerpo lógicamente cerrado de conceptos generales con referencia empírica e interrelacionados entre sí. A diferencia de la teoría — conformada por conceptualizaciones generalizadas-, el sistema teórico tiende idealmente al cierre lógico; intenta alcanzar un estado tal de integración, que cada consecuencia lógica de cualquier combinación de proposiciones del sistema se enuncie explícitamente en alguna otra proposición del mismo sistema (Parsons, 1967). Su función principal es la formulación y concatenación lógica de las proposiciones sobre hechos empíricos, con base en la observación y verificación.

Si se relacionan los planteamientos epistemológicos y lógico-metodológicos, puede verse que, lejos de cualquier duda ontológica, Parsons afirma la existencia real del mundo fenoménico, un universo que no es creado por la voluntad de los seres humanos, ni reducible a construcciones ideales derivadas de esquemas filosóficos. No obstante, en contraste con el empirismo, la ciencia no aprehende directamente ese mundo, puesto que al tratar con hechos (enunciados acerca de fenómenos), éstos se hacen visibles una vez efectuado un recorte de la realidad. El marco de referencia sirve a esos fines: delimita el campo de observación e ilumina aquellos aspectos relevantes analizados y explicados mediante el sistema teórico. Por consiguiente, el marco de referencia construye metodológicamente la realidad concreta que observa el sistema teórico; éste, a su vez, lleva a cabo la ordenación racional objetiva de dicha realidad. De este modo, el realismo analítico propone una explicación parcial de la realidad concreta; la descripción completa de un hecho empírico requiere más de un marco de referencia ${ }^{8}$ (Almaraz, 1981).

Si bien la distinción de etapas por las que atraviesa la TGA ${ }^{9}$ puede hacer perder de vista los aspectos que le otorgan unidad — principalmente, acelerar el proceso de maduración de la disciplina y da respuesta al problema de cómo es posible el orden social-, constituye un ejercicio fructífero al momento de identificar las transformaciones acaecidas en su interior. En esta línea, destacan tres momentos, cuyo principio de diferenciación es el carácter que adquiere el sistema teórico en cada uno: la teoría voluntarista de la acción — desarrollada en (Parsons, 1971a, 1971b)—, el modelo trisistémico — planteado en (Parsons, 1976a; Parsons \& Shils, 1968) — y el esquema AGIL —a partir de (Parsons, Bales, \& Shils, 1953)— (Giordano, 2015). Para los fines de este ensayo, cabe posar la mirada en el último período dado que en allí la TGA adquiere sus rasgos definitivos: el esquema AGIL se orienta a detallar la función imprescindible de cada

8 Básicamente, Parsons reconoce la existencia de dos marcos de referencia: el de la acción y el espacio-temporal. Para demostrar su diferencia, recurre al ejemplo del suicidio: ante un actor que salta desde un puente, el físico se pregunta por la velocidad de la caída, el tiempo que tarda el cuerpo en impactar en el suelo, la localización espacial del suceso, la dirección del movimiento, etc.; el científico social, en cambio, trata de especificar el fin de ese acto - la previsión del actor sobre su propia muerte_- el medio empleado — sabe que si salta, morirá - las condiciones — la consideración de una altura suficiente como para garantizar el cumplimiento de su cometido- Si para el físico la cuestión problemática es el "suceso", para el sociólogo el problema es el "motivo", y en ambos casos, lo no problemático constituye un dato (Parsons, 1971a).

9 Para citar algunos ejemplos de interés, Gerhardt (2002), interesado en los aspectos políticos, distingue cuatro etapas concordantes con La estructura de la acción social, los ensayos empíricos elaborados entre 1938 y 1945 , El sistema social y la teoría de los medios de intercambio, principalmente la tematización sobre el poder. Almaraz (1981), en cambio, reconoce tres etapas — subjetivista-idealista, analítica social-psicologista y objetivo-analítica - todas ellas conducentes a la elaboración de un metalenguaje. Bershady (1973) asevera que aún cuando la teoría experimenta variaciones, la postura epistemológica se mantiene, de modo que no hay por qué diferenciarla en períodos. 
subsistema para el mantenimiento de los límites del sistema general de acción ${ }^{10}$. Lo propio de este nuevo período consiste en la preponderancia que adquieren los problemas funcionales y, consecuentemente, su resolución, ligada a las condiciones de mantenimiento y desarrollo de los límites del sistema durante procesos de intercambio de inputs y outputs con el ambiente. En principio, un sistema refiere a relaciones de interdependencia entre sus partes 0 variables; esto es, la existencia de relaciones determinadas (no azarosas) que indican un orden de las conexiones entre sus elementos (Parsons, Bales, Shills, 1953). De esta manera, la definición se inscribe dentro de la Teoría General de Sistemas inaugurada por von Bertalanffy (1976). Así, los sistemas permanecen a lo largo del tiempo a condición de que mantengan su equilibrio: en caso de no satisfacer las exigencias de un contexto signado por el cambio constante, el sistema se disuelve, y se produce un fenómeno análogo a la muerte de los organismos biológicos. Dado que se trata de sistemas abiertos a un ambiente con el que traban relaciones de intercambio, ese equilibrio depende del mantenimiento de los límites y, a su vez, ambos dependen del cumplimiento de una función específica (Parsons, 1974, 1976b). Aquí, Parsons es explícito en señalar la influencia de la idea de homeostásis de Walter B. Cannon (Parsons, 1961).

En consecuencia, la acción social —principal objeto de estudio de la TGA — mantiene los límites con el ambiente siempre que sus cuatro subsistemas satisfagan una función particular: el organismo conductual, se relaciona con la adaptación, el subsistema de la personalidad con el logro de metas, el subsistema social con la integración y el subsistema cultural con el mantenimiento de pautas. Para el caso específico de la sociología, su objeto es el sistema social, núcleo integrativo de la acción. Para analizarlo, la tarea consiste en aplicar el AGIL

10 A su vez, vale remarcar que en el último período de su obra, también se observa un nuevo desplazamiento en lo que respecta a su postura metodológica: la teoría voluntarista de la acción proponía una metodología estructural, el modelo trisistémico, en cambio, se corre hacia el estructural-funcionalismo y el esquema AGIL, finalmente, se constituye en base a un análisis funcional. a su interior, por lo que se obtienen los siguientes subsistemas: la economía — ligada a la función de adaptación—, la política — vinculada al logro de metas_, la comunidad societaria — relacionada con la integración — y el sistema fiduciario — referido al mantenimiento de pautas-.

A la luz del esquema AGIL, su postura epistemológica sufre leves modificaciones: en (Parsons, 1961), el realismo analítico es repensado al interior de una de las dos dimensiones de orientación externa de los sistemas culturales, la que refiere a las categorías de significación de los objetos - la restante es la de las pautas de orientación hacia los objetos-. Precisamente en esta dimensión, al indagar acerca del campo propio de la metodología de la ciencia, se aduce que consta de cuatro componentes, que se relacionan con las cuatro funciones esenciales: la función adaptativa es cumplida por los hechos, que son enunciados sobre fenómenos empíricos. Nuevamente son diferenciados de los fenómenos, que si bien son objetos a los cuales se orienta el saber científico, no forman parte de los significados del objeto, y por ello, se encuentran fuera de los límites del sistema cultural. En cuanto al logro de metas, la tarea recae sobre la solución a los problemas empíricos, donde los hechos son interpretados más allá de su validez, ya que se organizan en problemas que tienen relevancia científica. Entre sus características, sobresale la de poder determinar nuevos hechos que no han sido contemplados por el sistema teórico, y así subsanar grietas en el conocimiento. Ahora bien, los problemas tienen relevancia porque se encuentran dentro de un sistema teórico, que es el tercer componente y se encarga de la función integrativa. Su trabajo consiste en poner en relación los distintos hechos dentro de un cuerpo de conocimiento común, ya que "los hechos no hablan por sí mismos, deben estar organizados" (Parsons, 1961: 965; traducción propia). A su vez, el sistema teórico sólo puede tener sentido cuando depende del último componente, el marco de referencia, encargado del mantenimiento de patrones. Un marco de referencia son premisas de un orden más elevado y se compone de conceptos primitivos que no se encuentran sujetos a validación empírica y que subyacen a los significados de los problemas que son investigados. 
A pesar de que la clasificación no presenta novedad $^{11}$, sí lo hace el establecimiento de una doble relación jerárquica entre sus componentes, idea tomada de la cibernética de Norbert Wiener ${ }^{12}$ (Parsons, 2007): una jerarquía de factores de control de carácter descendente, ordenada desde los subsistemas poseedores de mayor información hacia los que poseen menos (L-I-G-A). En este caso, el marco de referencia controla al sistema teórico —limita su campo de observación-; los problemas son relevantes porque el sistema teórico los define; y por último, se distingue entre fenómeno y hecho en virtud del problema empírico seleccionado. La segunda jerarquía concierne a los factores de condicionamiento y asciende desde los subsistemas que poseen mayor energía, hacia los que poseen menos (A-G-I-L): un problema que no se resuelve sobre la

11 Una visión diferente tiene Habermas, para quien la última etapa supone una ruptura implícita con el realismo analítico, el que pasa a ocupar un lugar retórico dentro de su esquema. El marco de la teoría, ahora, puede caracterizar sistemas que mantienen el equilibrio, de modo tal que es posible establecer modelos que simulen fragmentos relevantes de la realidad. Entonces, los enunciados sobre las normas, los valores, los fines y los recursos lograrían dar cuenta de relaciones empíricas. Con ello, la nueva versión adquiere un componente esencialista, pues "Io que antes había sido entendido como una proyección constructiva del científico cobra ahora connotaciones de una reconstrucción de las características de sistemas de acción que se estructuran a sí mismos" (Habermas, 1987: 338).

12 Junto a Rosenblueth, Wiener es considerado el fundador de la cibernética. Ambos construyen una red conceptual común para las problemáticas relacionadas con el campo de la ciencia del control y la comunicación en el animal y la máquina (von Foerster, 2006). Wiener trabaja en el desarrollo de máquinas autorreguladoras - es decir, máquinas que, dado un objetivo, son capaces de corregir su funcionamiento para alcanzarlo-. La elaboración del principio de oscilación —máquinas capaces de corregir su curso, pero no de alcanzar la meta, con lo que oscilan en torno a ella- acerca su investigación a los propósitos de Rosenblueth, quien en el campo de la biología busca comprender las alteraciones neurológicas denominadas temblores intencionales. Los dos desarrollan una red conceptual articulada en torno de la noción de circularidad, que abarca tanto a los sistemas artificiales como a los naturales; más tarde, la denominan cibernética (Pakman, 2006). base de hechos válidos no puede ser considerado científico; lo mismo sucede con una teoría que no se haya validado por la resolución de problemas de raíz empírica; finalmente, un marco de referencia que carece de utilidad para elaborar teorías que se relacionan con problemas empíricos, no tienen lugar dentro del campo de la ciencia.

\section{EL CONSTRUCTIVISMO OPERATIVO DE NIKLAS LUHMANN}

Desde la publicación de Sistemas Sociales (Luhmann, 1998b), Luhmann evidencia el pasaje de un modelo de irreductibilidad de lo social basado en la acción a uno asentado en la comunicación (Pignuoli 0campo, 2012), donde la idea de complejidad comienza a ocupar un lugar clave. Por considerarla una infinitud inobservable, la complejidad representa el mayor nivel de abstracción sobre el cual edifica su Teoría General de Sistemas Sociales. La complejidad lo abarca todo, más allá de ella no hay nada; al ser una unidad de referencia carente de límites, se convierte en un problema sin solución. Ante tal panorama, la única posibilidad de abordarla es desplazar su problematización; es decir, reducir complejidad. Ésta figura, exteriorizada como el horizonte de todas las posibilidades, obliga a aquel que se disponga a observarla, a efectuar una selectividad forzosa. El mecanismo que reduce complejidad y constituye el punto de partida de la Teoría General de Sistemas Sociales, es precisamente el concepto de sistema:

Las siguientes reflexiones parten del hecho de que existen sistemas ${ }^{13}$; no inician, por consiguiente,

13 Según Pignuoli Ocampo (2016) la doctrina de la emergencia es el basamento de ésta afirmación con la que Luhmann establece el punto de partida de su postura ontológica. Un orden de complejidad reducida —un sistema - implica un orden de emergencia, caracterizado por la "aparición de entidades y propiedades cualitativamente novedosas dentro del mundo, cuyas propiedades no se derivan ni están presentes en sus precursores ni en sus condiciones de posibilidad" (Pignuoli Ocampo, 2016: 159). Para el autor, el postulado luhmanniano según el cual las propiedades emergentes suponen al mundo, no lo crean, lo libera de las objeciones típicas que acusan a los emergentistas 
con una duda teórica del conocimiento... El concepto de sistema designa lo que en verdad es un sistema y asume con ello la responsabilidad de probar sus afirmaciones frente a la realidad (Luhmann, 1998b: 37).

El sistema construye su propia complejidad cuando establece los límites con su entorno, en una operación que le es exclusiva y gracias a la cual produce y reproduce por sí mismo los elementos que lo componen. De tal forma, el enlace selectivo sitúa, cualifica y establece las relaciones entre los elementos constitutivos del sistema y organiza su propia complejidad (Luhmann, 1998a, 1998b).

La existencia fehaciente de sistemas es sólo el cimiento sobre el cual se construye una teoría que sostiene, además, que los sistemas son autorreferenciales y autopoiéticos (Luhmann, 1998b). En sentido general, la autorreferencia se liga de modo directo con la diferencia entre sistema y entorno: los sistemas autorreferenciales entablan relaciones consigo mismos al trazar un límite con el entorno. La conservación del límite es indispensable para la conservación del sistema; sin límite, no hay autorreferencia. En busca de mayor precisión, surge la pregunta acerca de cómo es que el sistema logra mantener sus límites; y la respuesta, asevera que el sistema se vuelve a sí mismo sistema al enlazar operaciones propias con operaciones propias. En la medida en que logra enlazar la operación que le da identidad, continúa diferenciándose de su entorno — sigue existiendo-. Entonces, es la operación, el presupuesto para la existencia del sistema mismo, donde operación refiere a la reproducción de un elemento de un sistema autopoiético con base en los elementos del mismo sistema. Autopoiesis significa la determinación del estado siguiente del sistema a partir de la limitación anterior a la que llegó la operación. De esta manera, los sistemas se caracterizan por producir y reproducir por sí mismos los elementos que lo constituyen. Finalmente, dado que

de creacionistas y misticistas. Dichas apreciaciones le permiten concluir que, en el caso de Luhmann, el alejamiento de la tradición de pensamiento occidental no acarrea desechar el establecimiento de una postura ontológica con la cual elaborar premisas sobre la estructuración de lo real. las operaciones constituyen el presupuesto para las operaciones futuras, se concluye que los sistemas se encuentran clausurados operacionalmente; es decir que son autónomos respecto a su entorno. En esta línea, cabe aclarar que autonomía no implica independencia: "un sistema no puede existir sin entorno" (Luhmann, 1998: 15). El entorno es condición previa para la emergencia de un sistema autorreferencial —establecimiento de los límites-y para su conservación mediante el restablecimiento de energía e información — mantenimiento de los límites-. Lo que sí es independiente es la forma en que cada uno maneja su complejidad: complejidad reducida — sistema — y complejidad sin límite - entorno-.

Así, el proyecto de Luhmann propone la renovación del enfoque sistémico ${ }^{14}$. Si bien reconoce en la figura de Parsons un pionero en la aplicación de la idea de sistemas al estudio de las ciencias sociales, remarca la obsolescencia de su modo de emplearlo. En su óptica, Parsons utiliza dos modelos característicos de la tradición sistémica: por un lado, el de sistema en estado de equilibrio —que presupone la distinción entre estabilidad y perturbación-; por el otro, el de sistemas abiertos a un medio ambiente con el que mantienen relaciones de intercambio de inputs-outputs. A la luz de las críticas que han recibido ambos modelos, enfocadas a destacar su raíz tecnocrática y su preferencia por la estabilidad, Luhmann entiende que ninguna logró descubrir su verdadero defecto: no explicita qué es un sistema (Luhmann, 2007). Para rellenar el bache teórico, se vale de los principios de Autorreferencia, autopoiesis y clausura operativa, propios de una nueva generación de teóricos sistémicos entre Ios que destacan Heinz von Foerster, Gotthard Gunther y Humberto Maturana (Luhmann, 2007).

14 Vale destacar que la teoría de sistemas, tal como la entiende Luhmann, no constituye un campo unificado. El criterio es utilizado para englobar un conjunto de investigaciones que apuntan a temas similares y que comparten ciertos principios (Luhmann, 2007). Dado que el objetivo del presente trabajo no se centra en la teoría de sistemas, basta con señalar que bajo dicha nominación Luhmann introduce, principalmente, diversos aportes de la teoría general de sistemas, la cibernética y la teoría de la complejidad. 
Sobre tales cimientos erige una postura constructivista operativa, donde la diferencia entre operación y observación constituye uno de sus pilares. Sobre la primera, ya fueron adelantadas algunas especificaciones y se espera continuar con la tarea. Ahora, es preciso centrarse en la observación. En primer lugar, es una operación que lleva a cabo la diferencia entre indicar y distinguir. Según la lógica matemática de Spencer Brown, una forma traza una distinción que divide el universo de posibilidades en dos lados, de modo tal que no es posible estar en ambos a la vez. La forma, entonces, es un límite, una frontera que diferencia una parte indicada de otra que no lo está (la distinguida), haciendo imposible el paso de una a otra sin cruzarla. Observar, entonces, es indicar diferenciando un lado de la forma. Si se tiene en cuenta la dimensión temporal, cabe destacar que los dos acontecimientos son simultáneos —indicar es al mismo tiempo distinguir, así como también, distinguir es al mismo tiempo indicar-.

De esta diferenciación se desprende otra que divide, nuevamente, al universo en dos: no es lo mismo la realización de la observación de una operación que la observación de la operación como observación. Con respecto a la primera basta con observar lo que sucede. El problema surge cuando se intenta llevar adelante la segunda, ya que las operaciones realizan la reproducción del sistema de manera ciega. Esto quiere decir que una operación de observación no puede observarse a sí misma. Para la operación, su observación es un punto ciego, es decir que no puede observar su propia distinción. Para aclarar esta cuestión, es necesario referirse nuevamente a la dimensión temporal. Las operaciones son eventos que aparecen para desaparecer y no pueden ser repetidos, donde aparecer implica la posibilidad de ser señalado cronológicamente. La observación de una operación es un acontecimiento, un evento que sucede de manera temporalmente puntualizada y que inmediatamente se vuelve irreversible. Para observarse a sí misma debería disolver la simultaneidad operativa necesaria de operación y mundo, colocándose en una relación antes/después y, para ello necesitaría tiempo. En cambio, para la observación de una operación de observación hay que colocarse en un nivel de segundo orden. Es preciso observar — distinguir e indicar- cómo observa el observador observado; es decir, hay que observar mediante qué distinciones opera el observador de primer orden; de tal modo, surge un observador de segundo orden, quien, al volver reversible lo irreversible —el acontecimiento - está habilitado para llevar a cabo su historia, marcando un presente y un futuro.

Presentadas estas cuestiones, se procede a realizar un acercamiento a la concepción luhmanniana acerca de la realidad, y ulteriormente, a indagar su vínculo con la ciencia. En lo que respecta a la primera cuestión, el problema a resolver es por qué la observación es una operación; y luego ver cuál es la necesidad de diferenciarlas: en principio, la observación, es una designación distintiva. Indica un lado de la forma y discrimina el otro; 0 sea, traza una distinción. La particularidad radica en que su propia distinción es un punto ciego, no puede observarla ni diferenciarla, se encuentra latente - en este nivel, no logra autoobservarse-. Por ello, "es una operación” (Luhmann, 1996: 86). Una observación, utiliza su diferenciación, no la diferencia; para hacerlo, necesita poner en marcha otra operación de su propia observación, para lo cual, debería pasar el tiempo y actualizar un nuevo acontecimiento; es decir, realizar una observación de segundo orden. A fin de clarificar esta cuestión, remite a la comunicación, "la operación epistemológica básica", ya que en ella el problema se presenta de forma especialmente compleja (Luhmann, 1996: 87). La condición operativa de toda comunicación es la diferenciación entre información, acto de comunicar y comprensión. Si no se involucra a los tres, no hay comunicación, sólo percepción entre los presentes. El punto es que en una comunicación el hacer saber de las informaciones ocurre, diferencia y designa aquello que se informa; es decir que opera, y sólo por ello, la comunicación es una observación. Entonces, observar la operación comunicativa remite a lo tematizado: "operativamente siempre ocurre únicamente lo que ocurre". En este caso, "sólo se comunica lo que se comunica", Io designado (Luhmann, 1996: 88). Una vez que se observa la operación como observación se introduce el otro lado de la forma: se puede apreciar que la comunicación podría haber sido de otra manera y podría haber abordado otros temas. 
Una vez distinguidas, se afirma que la observación misma es una operación empírica, y por tanto, es real. El primer indicador de esta cuestión es que ella misma es observable: "la realidad está dada con la realización de la operación, y por lo tanto, todos los sistemas que observan son sistemas reales con sus correspondientes dependencias reales" (Luhmann, 1996: 62). Entonces, el punto de partida es la factibilidad empírica de la observación; y a su vez, el hecho de que la observación se encuentra empíricamente condicionada (Luhmann, 1996).

La radicalidad del constructivismo operativo acarrea el enfrentamiento con algunos postulados de la ciencia tradicional. Por caso, propone abandonar la clásica pretensión de objetividad: la garantía de que la observación es real es que es una operación; no la referencia a un mundo independiente, que garantice que si todos los observadores utilizan los mismos métodos se arribe a iguales afirmaciones sobre él. Al no existir un mundo libre de distinciones, se desecha la posibilidad de que un observador pueda contemplarlo desde fuera; y con ello, se acepta que toda observación altera al mundo en que se observa ${ }^{15}$.

15 Al reflexionar sobre estas cuestiones, Mascareño (2010) sostiene que en la postura luhmanniana es posible diferenciar dos niveles de mundo: un ultramundo y un mundo significativo. La existencia del segundo, sólo es posible a condición de que le anteceda el primero: para que emerja un mundo como horizonte de posibilidades de significación — compuesto por los sistemas que operan en el medio del sentido (sistemas psíquicos y sociales) — es necesaria una base material. Pese a ser inaccesible para la observación, el ultramundo es el mismo para todos; es decir que es empírico. La operación garantiza la materialidad del mundo sobre el cual se afirma la observación: "La operación es facticidad del ultramundo; la observación es validez del mundo para el observador. Dicho de otro modo, la operación es la ultrarealidad de la observación" (Mascareño, 2010: 12). Sobre ese sustento ultrarreal, avalado por la operación, se afirma lo real de la observación. Esta es libre de construir su validez, 0 sea, de indicar y distinguir aquello que pretenda indicar y distinguir. En conclusión, para Mascareño la desontontologización de la realidad encarada por esta postura no implica negar la realidad. Sin realidad, no habría operación, ni observación, ni distinción; y, si bien el mundo significativo no posee el estatus real del ultramundo, el ser al único mundo al que los sistemas que operan en el medio del sentido pueden acceder, se convierte en su mundo, en su realidad.
Ahora bien, el siguiente problema consiste en vincular esta postura con su concepción acerca de la ciencia: sobre la base de que una observación de observaciones sólo es posible si las observaciones por observar realmente ocurren -0 sea que las observaciones de primer orden son la condición de posibilidad de la existencia de las de segundo orden-, la ciencia es un tipo particular de observador que, producto de su diferenciación y evolución, se convierte en uno de los sistemas funcionalmente diferenciados de la sociedad moderna. Esto requiere una serie de especificaciones: en primer lugar, por el hecho de pertenecer al sistema social, el sistema científico opera en el medio de la comunicación; además, debido a que es un sistema funcionalmente diferenciado, es un sistema parcial de la sociedad encargado de reproducir una función específica, fundamental para continuar con la autopoiesis de la sociedad. Puntualmente, su función consiste en la construcción y obtención de conocimiento verdadero.

El conocimiento, condición para la ciencia, involucra dos distinciones. La primera atañe a la manera en que la comunicación expresa las consecuencias estructurales: si frente a una decepción las expectativas se mantienen se trata de un estilo normativo; al contrario, si las expectativas se corrigen, activa un estilo cognitivo. La segunda remite al problema de la adjudicación causal: si el cambio del estado del sistema se adjudica al entorno se habla de vivencia, si se adjudica al propio sistema, de acción. Sobre esta base, el conocimiento es "una observación condensada, y en un sentido más especial que presupone una capacidad evolucionada de diferenciación, una posición de expectativa como vivencia cognitiva" (Luhmann, 1996: 109). Lo propio de la ciencia, entonces, es que construye conocimiento en base a su propio código, el cual divide al universo comunicacional en dos: las comunicaciones verdaderas y las que no lo son. Por ello, para que la ciencia se consolide como sistema primero fue necesaria la evolución y diferenciación del medio de comunicación simbólicamente diferenciado verdad. Para distinguir conocimiento verdadero/no verdadero se asienta en sus propios programas que son las teorías y los métodos. Finalmente, a su interior la ciencia trabaja con conceptos — enunciados 
generados dentro del sistema- y no con hechos - enunciados del entorno- (Luhmann, 1996).

En suma, el sistema científico para continuar su reproducción opera en el medio verdad; a su vez, al desarrollar su función contribuye a la autopoiesis de la sociedad. Presentada esta cuestión, el constructivismo operativo es una comunicación situada en el sistema científico que, para garantizar su propia autopoiesis, debe enlazar comunicaciones que utilicen el código binario verdadero/no verdadero. En este marco, ¿por qué su postura epistemológica es constructivista? Porque se afirma que el conocimiento no se basa en su correspondencia con la realidad, sino en las construcciones de un observador. El constructivismo propone el abandono de la distinción clásica empírico/trascendental y la elección de la distinción operación/observación. En lugar de con objetos, trabaja con constructos, que son el resultado estructural de la autopoiesis del sistema científico, y no con instructos, que refieren a influencias del entorno. Entonces, el constructivismo es una autodescripción del sistema científico que observa la manera en que el sistema enlaza operaciones y continúa con su autopoiesis, en un entorno al que no conoce, sino que lo construye ${ }^{16}$. Se

16 El establecimiento como punto de partida de la existencia fehaciente de sistemas y la posterior elección de un marco constructivista abre interesantes líneas de investigación al momento de especificar la particularidad del constructivismo operativo. Solo para señalar algunas, con foco en la postura ontológica de Luhmann, Moeller (2012) argumenta que la radicalidad de su constructivismo se asienta en sus pretensiones realistas. Lo que la construcción construye es real; por lo tanto, constructivismo y realismo, lejos de excluirse, se implican. Para Moeller, el verdadero quiebre es con la ontología tradicional, que en base a la distinción ser/no ser, ha enfocado siempre en la unidad; en contraposición, entiende que la concepción luhmanniana es postmoderna, pues se ocupa de la diferencia: la realidad es irreductible a una sola forma de identidad, por lo que emerge una nueva ontología radical de la diferenciación — de múltiples realidades (la de cada observador), pero no múltiples mundos-. Finalmente, destaca que esta prioridad ontológica es el cimiento sobre el que Luhmann erige su postura epistemológica.

En una línea similar, Scholl (2012) sostiene que la epistemología de Luhmann oscila entre posiciones realistas y constructivistas. Afirma que ello no implica considera operativo porque la garantía de que dichas construcciones son reales, es la propia operación; por ende, la observación, al ser una operación, es real: sucede en el mundo fáctico. De este modo, optar por esta postura plantea el abandono de las preguntas del tipo ¿qué es lo que hay?, y pondera las preguntas acerca del cómo; así, la cuestión que debe resolver un observador de segundo orden consiste en averiguar “ ¿cómo construye un observador lo que construye para poder añadir otras observaciones?" (Luhmann, 1996: 51).

\section{EL VÍNCULO ENTRE REALIDAD Y CIENCIA EN EL REALISMO ANALÍTICO Y EL CONSTRUCTIVISMO OPERATIVO}

La exitosa recepción que tuvo La Estructura de la Acción Social inicia el proceso de consolidación del funcionalismo, con Parsons a la cabeza, como

una contradicción, dado que no se trata de puntos de vista incompatibles. Las pretensiones explícitamente científicas de la sociología de Luhmann lo enfrentan a posiciones anti-realistas escépticas 0 idealistas, frente a las cuales adopta un realismo débil. Esto se observa, principalmente, en el postulado fundacional de su teoría de sistemas "existen sistemas"; y también, en las distinciones medio/forma y observación de primer orden (realista)/segundo orden (constructivista). Por otro lado, entiende que su postura acerca de que la generación de conocimiento es relativa y relacional respecto al punto de vista del observador, lo convierte en un constructivista con más puntos en común que los aceptados con su variante radical.

En oposición, Nassehi (2015) desestima la oscilación señalada entre realismo y constructivismo y argumenta que el constructivismo operativo inaugura una tercera posición epistemológica. En base a la distinción entre operación/observación entiende que el postulado "existen sistemas" no se vincula con la distinción ontológica tradicional ser/no ser, ni tiene pretensiones metafísicas, sino que es una metáfora que indica que algo está operando: una observación empírica que presupone observaciones, y cuya prueba es, precisamente, la operación. En el caso de no aceptar este argumento circular que se justifica a sí mismo, el constructivismo operativo se desvanece: "Mi pregunta es ¿qué existe entre realismo y constructivismo? Mi respuesta: ¿existen? En realidad no (Sic!). Ambos operan" (Nassehi, 20015: 15). 
la corriente hegemónica dentro del campo sociológico. Su lugar central se mantiene incuestionado durante dos décadas, hasta que comienzan a generarse diversos ataques desde diferentes escuelas ${ }^{17}$ (Alexander, 2000; Fox, Lidz, \& Bershady, 2005; Ritzer, 1993). Entre las primeras voces que se alzan en su contra, sobresale la de C. W. Mills, quien en tono provocador, critica el elevado nivel de abstracción de sus principios. Según Mills, Parsons es el referente de un conjunto de pensadores preocupados por elaborar una gran teoría, que al mantenerse en un nivel de pensamiento demasiado general, inhabilita su puesta en práctica. Sus representantes, los grandes teóricos, devienen incapaces de descender desde las altas generalidades hacia los problemas reales y culminan fetichizando los conceptos y obstaculizando el desarrollo del deseado pensamiento sistemático (Mills, 1964).

Fue planteado que la postura epistemológica de Parsons trabaja con hechos, que no son los fenómenos en sí, sino afirmaciones sobre ellos, siempre realizadas sobre el recorte previo del marco de referencia. Por ello, el nivel de abstracción de sus lineamientos, más que una crítica es una constatación. El realismo analítico no busca matizar el carácter abstracto de sus enunciados; asume que se limita a observar el mundo transfenoménico. Para posicionarse de tal manera, primero se asevera que existe una realidad, un orden fáctico que trasciende al accionar humano. Se la considera inaprensible en toda su plenitud, razón por la cual el marco de

17 Pese a que la multiplicidad y heterogeneidad de las voces reaccionarias dificulta su reconstrucción, es posible citar algunos ejemplos de interés, que en mayor o menor medida dieron forma al movimiento crítico: en Estados Unidos sobresalen la teoría del conflicto — cuyos principales referentes fueron Coser, Rex y Dahrendorf-, la teoría del intercambio de Homans, el interaccionismo simbólico de Blumer y Goffman, la fenomenología de Shutz, la etnometodología de Garfinkel y la sociología cultural de Geertz; en Europa, la teoría crítica de Marcuse, la sociología histórica de Bendix y Mooser, y con ataques puntuales, Lackmood apuntó contra el lugar relegado del conflicto, Touraine a su idea de clase social, y Giddens y Bourdieu, a la escasa influencia del individuo en su teoría; a la extensa lista se suma la teoría de la dependencia, con origen en Latinoamérica, cuyos máximos referentes fueron Cardozo y Faletto (Alexander, 2000; Habermas, 1987; Sidicaro, 1992). referencia la recorta y construye metodológicamente el campo de observación del sistema teórico. Éste se encarga de ordenar racionalmente los hechos, que son representaciones ideales, no directas, de los fenómenos. En suma, el realismo analítico postula una representación adecuada (no literal) a lo real, basada en la correspondencia entre el orden lógico y el orden fáctico; por ello, se coloca a un nivel intermedio entre la pura empiria y la irrealidad. En el caso de Luhmann, pese a lo que una lectura prematura podría señalar, su constructivismo operativo también se erige sobre la existencia de una realidad. La observación, al ser una operación, es real; sucede en el mundo real. A pesar de que el sistema científico trabaja con constructos, estos deben tener una base operativa anclada en el mundo real. Por tanto, la inobservabilidad instantánea de su propia operación para quien opera, demanda la colocación en un nivel de segundo orden, temporalmente posterior pero equivalentemente real, para observar su operación.

Ahora bien, afrontar el problema de la existencia de la realidad no es el principal objetivo de sus posturas epistemológicas, más bien lo es el de establecer su vínculo con el sistema científico. Según Parsons, la ciencia recorta la realidad, iluminando aquellos aspectos que pretende estudiar. El marco de referencia define el campo de observación del sistema teórico, encargado de la formulación y concatenación lógica de los problemas que serán contrastados con los hechos. Para Luhmann, el recorte de la realidad depende del observador. Por ejemplo, el sistema científico indica una parte de la realidad al operar en el medio verdad: sólo reproduce comunicaciones codificadas en términos verdadero/no verdadero; pero a diferencia de la postura parsoniana, el objeto de estudio no está supuesto sólo como un correlato en la formación analítica de los hechos, sino como una totalidad del mundo referida a la diferencia entre sistema y entorno (Luhmann, 1998b); es decir que la ciencia no trabaja con elementos analíticos, lo hace con observaciones que no suponen más que diferencias.

Por otro lado, si el realismo analítico declara abiertamente ser una teoría abstracta, en el caso del constructivismo operativo tal caracterización no es tan nítida: por un lado podría sostenerse, si se foca- 
liza en que si bien la observación de la operación es la única forma de dar cuenta de ésta, nunca lo hace de forma plena; 0 sea que, las observaciones de segundo orden no tienen acceso total a la operación; su descripción siempre depende del observador. La operación, en su plenitud, es indescriptible. Además, sobre la base de que "el observador del observador no es un observador mejor: es tan solo uno más." (Luhmann \& De Giorgi, 1993: 445), se resalta que su única ventaja consiste en ofrecer más de lo que abarca la observación primaria. Pero por otro lado, el constructivismo operativo deja entrever que en el caso de que las verdades de la ciencia se consideren abstractas, lo mismo vale para el dinero que se utiliza para reproducir el sistema económico y para el poder que se emplea en el sistema político. La ciencia, entonces, es un observador más de la sociedad, imposibilitado de observar su operación al igual que el resto de los sistemas funcionalmente diferenciados que contribuyen a la continuidad autopoiética de la sociedad.

\section{CONCLUSIONES}

El objetivo del presente trabajo consistió en comparar las posturas epistemológicas de Parsons y Luhmann a fin de aportar al esclarecimiento del vínculo entre los autores en un área llamativamente poco frecuentada, dada su potencialidad de entrelazar sus respectivas concepciones acerca de la realidad y la manera en que la ciencia debe lidiar con esta. El resultado obtenido es que, en el caso de Parsons, el nexo entre realidad y ciencia son los hechos, que son afirmaciones sobre los fenómenos de carácter abstracto debido a que dependen del previo recorte del marco de referencia. La ciencia, entonces, puede aprehender lo real pero siempre por medio de elementos analíticos. Con respecto a Luhmann, las observaciones de segundo orden posibilitan observar las operaciones, que son reales, como observación. Específicamente, la ciencia observa la realidad a través del medio de comunicación simbólicamente generalizado verdad, diferenciado a lo largo de la evolución y catalizador del sistema científico.

A su vez, el resultado de la comparación permite iluminar una nueva clave interpretativa acerca de la conexión entre los autores estudiados. Para presentarla, vale volver a observar por última vez, cómo emplean la idea de sistema: afín a su epistemología, el concepto parsoniano de sistema es considerado un elemento analítico. Con respecto a la especificación de sus propiedades -interrelación de sus elementos, equilibrio y mantenimiento de límites frente a un ambiente con el que entabla relaciones de intercambio - se encuentra fuertemente influenciada por la primera camada de pensadores sistémicos $^{18}$ —von Bertalanffy, Wiener y Cannon, principalmente- (Camou, 1997; Niklas Luhmann, 2007). A los ojos de Luhmann, si bien reconoce la importancia que tuvo Parsons al momento de colocar esta idea en el centro del interés científico, la evolución al interior de la teoría de sistemas da un giro fundamental con el que pretende trascender la clásica distinción filosófica entre trascendentalismo y empirismo. Lo común a estas corrientes, afirma, es el presupuesto sobre el que asientan sus conceptualizaciones: la externalidad del observador. Mientras que el trascendentalismo concibe que los conceptos (a priori) atraviesan la realidad; el empirismo, en contraste, supone que los sistemas se encuentran constituidos en la realidad, por lo que el trabajo teórico se limita a su descripción tal cual son. Para Luhmann, el principal hallazgo de la nueva generación de teóricos sistémicos es el de liberarse de dicho presupuesto al aceptar las implicaciones autológicas de toda observación: no hay observador externo, o sea que, "lo que es válido para los objetos es también válido para el observador" (Luhmann, 2007). De esta manera, al interior de la teoría de sistemas hay un desplazamiento de la tradicional concepción en base a las relaciones que se establecen entre sus elementos (von Bertalanffy, 1976), a una donde la operación propia es la que permite establecer la diferencia entre sistema y entorno (Luhmann, 1998b). Esto debe combinarse

18 La denominación hace referencia a aquellos autores que intentaron situar en el centro del debate científico a la idea de sistemas, donde sobresale la figura de von Bertalanffi. Por supuesto, esto no significa que hayan sido los primeros en incorporar al sistema dentro de sus conceptualizaciones; por ejemplo, Camou rastrea su utilización desde Aristóteles hasta la actualidad (Camou, 1997). 
con el viraje de una cibernética de los sistemas observados — como la de Wiener - a una cibernética de los sistemas observantes, cuyo punto de partida es que "todo lo dicho es dicho por un observador" (von Foerster, 2006: 89).

Esta constatación permite arribar a una última conclusión: el constructivismo operativo puede leerse en clave de una propuesta que pretende superar las aporías intrínsecas al realismo analítico. Al referirse a la TGA, Luhmann sostiene que "la teoría deja abiertas las cuestiones de la autoimplicación cognitiva, ya que no dice mucho sobre el grado de congruencia entre la conceptualidad analítica y la formación real de los sistemas" (Luhmann, 2007: 53). Sobre ese punto se asienta su principal defecto: no logra dar el giro decisivo capaz de entrelazar teoría de la sociedad con teoría de sistemas. El realismo analítico, entonces, no concibe que el propio conocimiento de los sistemas sociales se encuentra atado a las condiciones sociales de las que emerge (Luhmann, 2007); es decir, continúa elucubrando dentro del supuesto de la externalidad del observador. Para liberarse del callejón sin salida al que arriba esta concepción, propone dejar de concebir al sistema como un hecho - un elemento analítico construido por la ciencia e iluminado por su marco de referencia específico - y partir de la afirmación fehaciente de su existencia. Sobre esta base, desarrolla una postura que es constructivista porque considera que el conocimiento generado en el campo de la ciencia es construido por un observador. Además, el criterio de operatividad le permite anclar su epistemología en la realidad. Así, su teoría del conocimiento lo distancia de posturas de corte escéptico, como el "constructivismo radical" (Glasersfeld, 1994). Si bien comparte su pretensión de radicalidad, abandona la referencia al sujeto y coloca en su lugar a un sistema empíricamente observable, clausurado operativamente y autorreferencial: el social (Luhmann, 1990b; 1998a). Por ultimo, en comparación con el realismo analítico, el constructivismo operativo postula una relación más directa con lo real, pues no está mediada por elementos analíticos.

En otro orden, Luhmann aduce que optar por un constructivismo operativo acarrea un desplazamiento de las preguntas sobre el qué a las del cómo. Luego del trabajo realizado, se sostiene que tal decisión, en lo que respecta al posicionamiento epistemológi$c 0$, puede ser aplicada a ambos casos. El enfoque en el vínculo entre ciencia y realidad prima por sobre la evaluación de lo real. Los dos autores afirman su existencia, que es el telón de fondo sobre el cual edifican sus teorías; sin embargo, el principal interés no es qué es lo real sino cómo la ciencia trabaja con ese trasfondo. Mientras Parsons opta hacerlo mediante elementos analíticos, Luhmann adopta las observaciones de segundo orden.

Para finalizar, se dijo que el análisis efectuado podía aportar a la clásica discusión acerca de los límites de la sociología. En esta línea, cabe decir unas palabras acerca de la relación entre las respectivas posturas epistemológicas de los autores seleccionados y el área en la que se especializaron dentro del campo de la ciencia: la sociología.

En el caso de Parsons, hay una clara delimitación de tareas. Primero elabora una postura epistemológica para definir el estatus de los conceptos respecto a lo real. Una vez propuesto el realismo analítico, sus sucesivos trabajos se enfocan en el desarrollo del sistema teórico que fomente la maduración de la disciplina. De esta manera, todos los elementos analíticos que la TGA propone — sistema, acción, sociedad, orden, integración, etc.- - se mantienen en el plano transfenoménico: son hechos; 0 sea afirmaciones sobre fenómenos siempre realizadas sobre el recorte previo del marco de referencia de la acción. Para Luhmann, en cambio, epistemología y teoría de la sociedad están en una relación circular. Su apuesta es definir la epistemología sobre la noción de observador en lugar de la tradicional distinción sujeto/objeto. Una epistemología centrada en el observador implica seleccionar el sistema de referencia —organismos, sistemas psíquicos 0 sistemas sociales-. La teoría de la sociedad es una observación de segundo orden acerca de cómo observa la sociedad, un tipo particular de sistema social; se trata de una descripción sociológica de la sociedad en la sociedad, y por lo tanto, de una autodescripción. Así, las implicancias epistemológicas están incorporadas en la teoría de la sociedad o en otras palabras, la teoría de la sociedad se encuentra epistemológicamente informada.

En conclusión, a fin de cimentar sus teorías sobre sólidas bases teóricas, ni Parsons ni Luhmann 
eluden la problematización de cuestiones pertenecientes al campo de la epistemología. Por ello, sus reflexiones invitan a repensar los límites de la disciplina con respecto al resto de las áreas del saber, un ejercicio que además del enriquecer a la propia sociología, estimula al conjunto del sistema científico.

\section{AGRADECIMIENTOS Y FINANCIACIÓN}

Facultad de Ciencias Sociales de la Universidad de Buenos Aires, Buenos Aires, Argentina.

N. ${ }^{0}$ resolución de otorgamiento de la beca: 7117 $/ 13$.

\section{REFERENCIAS BIBLIOGRÁFICAS}

Alexander, J. C. (2000). Las teorías sociológicas desde la segunda guerra mundial. Análisis multidimensional. Barcelona: Gedisa.

Almaraz, J. (1981). La teoría sociológica de Talcott Parsons. Madrid: Centro de Investigaciones Sociológicas.

Bershady, H. J. (1973). Ideology and Social Knowledge. Oxford: Blackwell.

Camou, A. (1997). En torno al concepto de sistema social: Pareto, Parsons, Luhmann. En A. Camou \& C. M. J. Esteban (eds.), La sociedad compleja. Ensayos en torno a la obra de Niklas Luhmann (pp. 83-112). México: Triana Editores.

Castro Muzupappa, J. E. (1997). Niklas Luhmann y la tradición sociológica. In A. Camou \& C. M. J. Esteban (eds.), La sociedad compleja. Ensayos en torno a la obra de Niklas Luhmann (pp. 51-82). México: Triana Editores.

Chernilo, D. (1999). Integración y Diferenciación. La teoría de los medios simbólicamente generalizados como programa progresivo de investigación. Cinta de Moebio. Revista de Epistemología de Ciencias Sociales, (6), 313-405.

Fox, R., Lidz, V., \& Bershady, H. J. (2005). Introduction. In R. Fox, V. Lidz, \& H. J. Bershady (eds.), After Parsons: A Theory of Social Action for the Twenty First Century. New York: Russell Sage Foundation.
Fuchs, S. (2001). Networks and Systems. En J. Treviño (ed.), Talcott Parsons Today. His Theory and Legacy in Contemporary Sociology (pp. 129-139). Lanham (Maryland): Rowman \& Littlefield Publishers, INC.

García Ruiz, P. (1997). Los medios simbólicos, ¿de comunicación o de intercambio?: el legado parsoniano en Luhmann. Revista Anthropos: HueIlas Del Conocimiento, 173-174, 100-113.

Gerhardt, U. (2002). Talcott Parsons An Intellectual Biography. Cambridge: University Press.

Giordano, P. M. (2015). Avatares del cambio en la Teoría General de la Acción de Talcott Parsons General Theory of Action. Revista Sociológica. Departamento de Sociología de La Universidad Autónoma Metropolitana-Azcapotzalco, 30 (85), 197-232.

Habermas, J. (1987). Teoría de la Acción Comunicativa. Tomo II. Madrid: Taurus.

Izuzquiza, I. (2008). La sociedad sin hombres. Niklas Luhmann o la teoría como escándalo. Critica-Revista Hispanoamericana de Filosofia. España: Anthropos.

Lagunas, D. (2016). El legado del funcionalismo. Limitaciones teóricas y excesos etnográficos. Revista Espanola de Sociologia, 25 (2), 241-257.

Luhmann, N. (1973a). Función y Causalidad. En La Ilustración Sociológica y Otros Ensayos (pp. 9-47). Buenos Aires: Editorial Sur.

Luhmann, N. (1973b). Método Funcional y Teoría de Sistemas. En La Ilustración Sociológica y Otros Ensayos (pp. 48-91). Buenos Aires: Editorial Sur. Luhmann, N. (1990). ¿Por qué AGIL? Revista Sociológica. Departamento de Sociología de la Universidad Autónoma Metropolitana-Azcapotzalco, 5 (12), 377-401.

Luhmann, N. (1996). La Ciencia de la Sociedad. México: Anthropos.

Luhmann, N. (1998). ¿Cómo se pueden observar estructuras latentes? El Ojo del Observador. Contribuciones Al Constructivismo, 60-72.

Luhmann, N. (1998). Complejidad y modernidad: de la unidad a la diferencia. (J. Beriain \& J. M. García Blanco, Eds.). Madrid: Editorial Trotta.

Luhmann, N. (1998). Sistemas Sociales: Lineamientos para una teotía general. Barcelona: Anthropos. 
Luhmann, N. (2007). Introducción a la teoría de sistemas. México: Universidad Iberoamericana. Colección teoría social.

Luhmann, N., \& De Giorgi, R. (1993). Teoría de la Sociedad. México: Universidad Iberoamericana.

Mascareño, A. (2009). Medios simbólicamente generalizados y el problema de la emergencia. Cinta de Moebio, Revista de Epistemología de Ciencias Sociales, 36, 174-197.

Mascareño, A. (2010). Construct this! 0 por qué el constructivismo sistémico es real. Revista Mad. Magister En Análisis Sistémico Aplicado a La Sociedad, 23, 9-24.

Mills, C. W. (1964). La imaginación sociológica. México: Fondo de Cultura Económica.

Moeller, H.-G. (2012). The Radical Luhmann. New York: Columbia University Press.

Nafarrate, J. (2007). Introducción. En Introducción a la Teoría de Sistemas (pp. 7-19). México: Universidad Iberoamericana. Colección teoría social.

Pakman, M. (2006). Introducción. En H. von Foerster (ed.), Las semillas de la cibernética. Barcelona: Gedisa.

Parsons, T. (1961). An Outline of the Social System. In Theories of Society: Foundations of Modern Sociological Theory. Vol. I (pp. 30-79). New York: The Free Press.

Parsons, T. (1967). La posición actual y las perspectivas de la teoría sistemática en Sociología. En Ensayos de Teoría Sociológica. Buenos Aires: Paidós.

Parsons, T. (1971a). La Estructura de la Acción Social. Tomo 1. Madrid: Guadarrama.

Parsons, T. (1971b). La Estructura de la Acción Social. Tomo 2. Madrid: Guadarrama.

Parsons, T. (1974). El sistema de las sociedades modernas. México: Editorial Trillas.

Parsons, T. (1976a). El Sistema Social. Madrid: Alianza.

Parsons, T. (1976b). Sistemas sociales. En Enciclopedia Internacional de Ciencias Sociales (Tomo IX, pp. 710-721). Madrid: Ediciones Aguilar.

Parsons, T. (2007). American Society. A Theory of the Societal Community. London: Paradigm Publishers.
Parsons, T., Bales, R., \& Shils, E. (1953). Working papers in the theory of action. New York: Free Press.

Parsons, T., \& Shils, E. (1968). Hacia una teoría general de la acción. Buenos Aires: Kapelusz.

Pignuoli Ocampo, S. (2012). Los modelos de irreductibilidad social en la teoría sistémica de Niklas Luhmann. Revista Española de Sociología, 17, 27-48.

Pignuoli Ocampo, S. (2016). Aportes de las teorías sociológicas a la discusión de la ontología. Los casos de Luhmann, Habermas y Latour. Revista de Filosofía (Madrid), 41 (1), 153-179.

Ritzer, G. (1993). Teoría Sociológica Clásica. Madrid: MacGraw-Hill.

Savage, S. (1999). Las teorías de Talcott Parsons. México: MacGraw-Hill.

Scholl, A. (2012). Between realism and constructivism?: Luhmann's ambivalent epistemological standpoint. Constructivist Foundations, 8 (1), 5-12.

Sidicaro, R. (1992). Las sociologías después de Parsons. Sociedad, Revista de La Facultad de Ciencias Sociales, UBA, 1.

Vanderstraeten, R. (2002). Parsons, Luhmann and the Theorem of Double Contingency. Journal of Classical Sociology Copyright, 2 (1), 77-92.

von Bertalanffy, L. (1976). Teoría General de Sistemas. Buenos Aires: Fondo de Cultura Económica.

von Foerster, H. (2006). Las semillas de la cibernética. Barcelona: Gedisa.

Whitehead, A. N. (1985). La Función de la Razón. Madrid: Tecnos.

\section{NOTA BIBIOGRÁFICA}

Pedro Martín Giordano es licenciado en Sociología de la Universidad de Buenos Aires, Argentina. Becario doctoral UBACyT en Ciencias Sociales, con sede de trabajo en el Instituto de Investigaciones Gino Germani de la Facultad de Ciencias Sociales de la Universidad de Buenos Aires bajo la dirección de la Dra. Perla Aronson. Principales áreas de trabajo: Teoría Sociológica, Teoría Social, Constructivismo. 